\title{
SIG NA INTERNET: EXEMPLO DE APLICAÇÃO NO ENSINO SUPERIOR
}

\author{
Alfredo Pereira de Queiroz Filho*
}

\begin{abstract}
Resumo: Este trabalho relata o uso do software SpringWeb no curso de Introdução à Cartografia, ministrado no primeiro semestre de 2002 aos alunos do Departamento de Geografia da Universidade de São Paulo, e aborda os principais aspectos da integração de dados que Ihe dão suporte. As possibilidades de trabalhar fora do horário e local de aula, associar os conceitos básicos de Cartografia e minimizar o investimento em software e hardware são consideradas as principais vantagens dessa aplicação. As desvantagens mais relevantes se referem ao heterogêneo conhecimento de informática dos alunos, à necessidade de uso de banda larga para acessar o exercício e à exigência de instalação - no primeiro acesso - de um plug-in (programa Java).
\end{abstract}

Palavras-chave: Sistema de Informações Geográficas (SIG); Internet; Cartografia; Integração de dados.

\section{Introdução}

0 pressuposto que orienta este trabalho é o de que, independentemente do grau de aperfeiçoamento e do tipo de utilização, a incorporação dos Sistemas de Informações Geográficas (SIG) pela comunidade acadêmica está consolidada. No entanto, o uso indiscriminado das técnicas de quantificação e o conseqüente empobrecimento da capacidade de reflexão, num passado recente, fomentou uma atitude de cautela em relação à aplicação de técnicas de análise espacial.

É possivel estabelecer uma série de semelhanças entre as técnicas quantitativas e os SIGs. De acordo com WATERS (1997), o ano de 1997 marca o fim da "revolução" dos Sistemas de Informações Geográficas. Para ratificar sua afirmação, o autor resgata o artigo de lan Burton, de 1963, sobre o término da "revolução" quantitativa na Geografia, e aborda aspectos positivos e negativos que constata serem comuns aos dois períodos. Os indícios que mostram o término do referido momento de transformação são:

- os conceitos consagrados incorporam as novas técnicas, idéias e procedimentos;

- os termos estatísticos e o uso das tecnologias tornam-se parte da rotina de pesquisadores, docentes, profissionais, alunos etc.;

- $\quad$ aumento do número de geógrafos que sentem necessidade de conhecer as técnicas;
- incremento do número de disciplinas, nos cursos superiores, ligadas aos métodos quantitativos;

- geógrafos, com distintas visões de mundo, comentam sobre a importância de métodos quantitativos.

Da mesma forma, as principais críticas ao uso da quantificação da década de 1960 são válidas para os SIGs ${ }^{1}$. As mais importantes são:

- a quantificação negligenciou outras ferramentas desenvolvidas e aperfeiçoadas pelos geógrafos;

- $\quad$ alguns aspectos estudados pela Geografia não podem ser quantificados;

- $\quad$ uso incorreto, inapropriado e indiscriminado de métodos estatísticos;

- $\quad$ arrogância de alguns pesquisadores que trabalham com "novas" tecnologias. Em determinados casos, valorizam mais a técnica do que o objeto estudado.

Um dos elementos que podem diferenciar as técnicas quantitativas e os SIGs é o grau de dependência do desenvolvimento tecnológico. A evolução dos SIGs está atrelada à progressão dos recursos da informática. 0 aumento da capacidade de armazenamento e processamento, a diminuição dos custos de hardware e software e, particularmente, a melhoria da interface com o usuário foram responsáveis diretos pela incorporação da tecnologia nos segmentos de pesquisa e ensino. Na visão de CÂMARA et al. (1996), é possível agrupar as transformações dos SIGs em três gerações distintas:

\footnotetext{
* Departamento de Geografia USP, C.P. 26097, 05513-970 São Paulo (SP), Brasil. e-mail: aqueiroz@usp.br

1 Mais recentemente, o National Center for Geographic Information and Analysis (NCGIA), da Universidade da Califórnia, por meio de sua Research Iniciative 19, de março de 1994, realizou uma reunião com especialistas para discutir como a população, espaço e ambiente são representados em um SIG.
} 
- a primeira, a do CAD cartográfico, enfatizava o mapeamento, ou seja, o processo de entrada dos dados sem a definição prévia de um esquema conceitual. Caracterizou-se pelo limitado suporte de bancos de dados e foi desenvolvida a partir do início da década de 1980;

- a segunda, a dos bancos de dados, é caracterizada por sistemas que operam como um banco de dados geográficos, em que os dados tratados possuem atributos descritivos e representação geométrica no espaço geográfico. Essa fase começou no princípio da década de 1990 e ainda está em desenvolvimento (banco de dados relacional);

- a terceira, a das bibliotecas geográficas digitais ou dos centros de dados geográficos, diferencia-se pelo fato de o banco de dados geográficos ser compartilhado por várias instituições. Os dados são armazenados remotamente e acessados via Internet ${ }^{2}$. 0 registro das atividades dessa geração data do final da década de 1990 (sistemas orientados a objetos).

Dentre os inúmeros desafios que surgiram, no bojo dessa última geração, o de maior destaque e complexidade é, indiscutivelmente, o processo de integração de dados. 0 termo que melhor qualifica o contexto é a padronização (dos dados e interfaces), e o objetivo principal é o estabelecimento de normas para o compartilhamento dos bancos de dados por meio da Internet. Sua motivação decorre da constante duplicação de esforços - e, conseqüentemente, desperdício de recursos - nas atividades de atualização das bases cartográficas e de dados, por causa da falta de intercâmbio entre as instituições.

É nesse contexto de evolução tecnológica e do seu respectivo processo de assimilação que o presente trabalho se insere. Seu objetivo é relatar o uso do SpringWeb em uma das atividades do curso de Introdução à Cartografia e abordar os termos, os conceitos e os aspectos da integração de dados mais relevantes.

\section{Integração de dados}

Integração, lato sensu, é o ato de integrar. Integrar, do latim integrare, significa tornar inteiro, completar, juntar, inteirar, reunir, combinar. A essência do processo de integração de dados, então, é a reunião de informações, em boa parte dos casos, oriunda de distintas fontes.

Embora o espírito desse processo possa sugerir uma idéia singela, quando se trata do ambiente digital e, sobretudo, da possibilidade de obter dados via Internet, surgem especificidades que tornam sua implementação nada elementar. Integração se torna sinônimo de padronização, o que implica na definição de formatos comuns de dados para milhões de usuários que possuem objetivos distintos, utiliza, pelo menos, uma dezena de tipos de "software SIG" diferentes e acessam a Internet com equipamentos, navegadores e velocidades diversos.

Assim, a tarefa de criar normas para a integração de dados é um empreendimento de vulto, que excede as possibilidades de atuação isolada de empresas, profissionais, acadêmicos ou experts. Conforme GREEN \&t BOSSOMAIER (2002), dois grandes grupos encamparam as atividades de padronização: o Object Management Group (OMG), associado à ascensão da tecnologia dos sistemas orientados a objetos ${ }^{3}$, e o OpenGis, que tem seu foco voltado para os Sistemas de Informações Geográficas.

0 consórcio OMG, fundado em 1989, é atualmente composto por cerca de 800 membros e, embora formado majoritariamente por empresas e indústrias privadas, não possui fins lucrativos. Seu objetivo principal é a especificação de um conjunto de padrões para um sistema de gerenciamento de objetos distribuídos. Suas atividades estão centradas no desenvolvimento dos padrões CORBA (Common Object Request Broker Architecture) e do protocolo IIOP (Internet Inter-ORB Protocol).

A principal organização que representa os interesses dos grupos que operam na área de geoprocessamento é o consórcio OpenGis. Fundado em 1994, reúne cerca de 220 entidades associadas (empresas, universidades, órgãos governamentais etc.) com o objetivo de criar especificações para a integração de informações espaciais. 0 consórcio OpenGIS e o Federal Geographic Data Committee (FGDC) trabalham em conjunto, desenvolvendo a especificação ISO/TC 211, para estabelecer um padrão formal e global de metadados espaciais.

0 FGDC é um comitê norte-americano, organizado em 1990, que coordena o desenvolvimento de uma infra-estrutura nacional de dados espaciais (National Spatial Data Infrastructure - NSDI). É responsável pela elaboração de políticas, padrões e procedimentos para que as organizações governamentais norteamericanas produzam e compartilhem, cooperativamente, dados geográficos. É composto por representações de 17 agências federais norte-americanas e recebe cooperação de organizações estaduais e locais, comunidade acadêmica e setor privado.

2 A definição de Internet mais difundida é a de uma rede mundial de computadores. Entretanto, será considerada como um sistema de redes interconectadas, que utiliza o protocolo TCP/IP (Transmission Control Protocol/ Internet Protocol). World Wide Web é a interface gráfica do usuário da Internet, que pode ser definida como uma grande coleção de documentos interligados por hipertexto, associados a diversos recursos de multimídia.

3 Nessa linguagem, o programa é construido em função dos dados a serem manipulados e dos métodos que controlam esses dados. Juntos, dados e métodos procuram simular o comportamento dos objetos do mundo real. 
Dentre as inúmeras atividades dessas instituições, que atuam combinada e simultaneamente no processo de integração de dados, deve-se ressaltar o desenvolvimento de um padrão de metadados e de procedimentos para a interoperabilidade dos sistemas. Esses dois aspectos e os repositórios de dados (data warehouse), resultantes desta evolução, serão abordados nos tópicos a seguir.

\section{Metadados}

De forma genérica, metadados podem ser definidos como dados sobre os dados. Representam o histórico do seu processo de obtenção/geração, uma vez que descrevem o seu conteúdo, sua qualidade, sua condição e demais características relevantes.

0 grande desafio para a criação dos metadados é estabelecer um padrão compacto e equilibrado para as diferentes necessidades dos usuários. Deve-se considerar a meIhor forma de representar o seu conteúdo e, simultaneamente, o custo e o tempo para a sua implementação.

O FGDC desenvolveu o padrão de conteúdo para metadados denominado Content Standard for Digital Geospatial Metadata (CSDGM). Ele possui 245 elementos, isto é, quesitos que contêm informações sobre os dados, distribuídos por 11 categorias (o preenchimento desses elementos pode ser obrigatório, obrigatório quando aplicável ou optativo $)^{4}$. Deve-se destacar, no entanto, que a complexidade e o custo de implementação do CSDGM levaram os usuários a criar o Metalite. Esse padrão é uma simplificação do CSDGM, composto somente pelos 29 quesitos obrigatórios.

Na opinião de LIMA (2002), o padrão do FGDC requer uma excessiva quantidade de informações, de aplicação questionável, exigindo o preenchimento de dezenas de formulários. A burocracia envolvida na sua adoção não se traduz em benefícios proporcionais.

Contudo, considerando-se a quantidade de bases cartográficas e de bancos de dados existentes, parte já disponivel na Internet ${ }^{5}$, a implementação de metadados é extremamente útil, pois permite, com a uniformização dos mecanismos de buscas, o acesso a um número muito maior de fontes de dados.

\section{Interoperabilidade}

Interoperabilidade é a capacidade de compartilhar e trocar informações de um sistema. Segundo GOODCHILD et al. (1997), os principais significados de interoperabilidade são:

- $\quad$ tornar aberta a estrutura interna dos arquivos de dados. Permitiria que os usuários desenvolvessem aplicativos que combinassem componentes de software de diferentes fabricantes, de forma a compor a melhor ferramenta para a sua necessidade;

- viabilizar a capacidade de conversão de dados entre os softwares. Como cada produto possui uma forma distinta de armazenar dados, significa que os usuários poderiam utilizar os seus dados em qualquer software, convertendo os diferentes tipos de arquivos para o formato desejado, sem que houvesse perda de informação;

- popularizar a interação com o usuário. Os projetistas dos sistemas desenvolveriam interfaces de forma que pudessem ser personalizadas, tornando-as mais familiares aos usuários.

Conforme LIMA (2002), a busca pela interoperabilidade é uma tarefa complexa, que vem sendo tratada em três diferentes frentes: a conversão entre formatos de dados específicos de cada SIG, a conversão entre semânticas de bancos de dados distintos e o desenvolvimento de modelos gerais de dados geográficos. 0 autor considera dois níveis de abordagem da interoperabilidade: sintático e semântico.

No nível sintático, o armazenamento dos dados geográficos é organizado em estruturas próprias que descrevem suas características. A conversão sintática direta realiza a tradução dos arquivos de informação geográfica entre diferentes estruturas lógicas de armazenamento de dados. Os dois principais procedimentos são:

- exportar no padrão ASCII, de fácil legibilidade;

- documentar as estruturas internas de dados (ex.: SHP, do ArcView).

0 nível semântico está relacionado à representação conceitual da informação geográfica presente em cada sistema. Como não há padronização, entidades iguais podem ser denominadas por nomes diferentes, ao passo que um mesmo nome pode ser utilizado para descrever entidades distintas em domínios diferentes. Para que haja êxito nessa operação, é necessário o compartilhamento de significado, isto é, a correspondência entre os conceitos de diferentes sistemas.

\section{Repositórios de dados (data warehouse)}

Segundo GREEN \& BOSSOMAIER (2002), data warehouse é uma coleção organizada de bancos de dados e processos que permite, via Internet, a recuperação, a interpretação e a visualização de informações. Esses repositórios podem ser

4 Para maiores informações consultar http://www.fgdc.gov/metadata/csdgm/ (acesso em 28 jan. 2003).

5 Deve-se destacar o site http://www.maproom.psu.edu/dew da Universidade da Pensilvânia, que possui mapas digitais, na escala 1/1.000.000, de todo o mundo. Para o Brasil, os temas de hidrografia, rodovias, ferrovias, curvas de nível, cidades e aeroportos estão disponíveis por estado. As informações estão nos formatos Arcinfo e ArcView (acesso em 17 fev. 2003). 
considerados como um resultado do processo de integração de dados, edificado sobre a padronização das estruturas e das interfaces. As suas características mais importantes são:

- conter vários bancos de dados, de organizações e fontes distintas;

- $\quad$ permitir consultas complexas, interpretação e visualização dos dados;

- possuir grande volume de dados.

Outra iniciativa correlata é a Clearinghouse (National Geospatial Data Clearinghouse). É utilizada para denominar um sistema descentralizado de servidores, conectados à Internet, que contêm informações descritivas dos dados geográficos. Essa iniciativa é patrocinada pelo FGDC, e adota o padrão ANSI Z39.50-1995 para a consulta, busca e apresentação dos resultados das pesquisas dos usuários.

Embora sua função não seja a de armazenagem física dos dados, indica ao usuário onde encontrar o melhor dado espacial para as suas necessidades, por intermédio de pesquisas sobre os metadados. Tal como um catálogo telefônico digital, informa onde encontrar os dados, em que local eles são produzidos e mantidos.

A expressão Geography network pode ser entendida como o nome comercial de uma Clearinghouse. Ela fornece a infra-estrutura necessária para permitir o compartilhamento das informações geográficas entre os produtores de dados, instituições governamentais e usuários de qualquer parte do mundo, pela Internet. É uma fonte on-line para busca e compartilhamento de informações geográficas.

\section{Considerações sobre a utilização da informática no ensino}

Nesse item serão mencionadas algumas das reflexões que surgiram no processo de elaboração da atividade didática. Dentre os aspectos gerais sobre o uso de computadores em sala de aula, os considerados mais importantes foram:

- conhecimento heterogêneo de informática dos alunos. A maioria dos estudantes já tinha familiaridade com os procedimentos, mas uma pequena parte não demonstrou que poderia acessar o site e elaborar os exercícios sem ajuda de terceiros;

- como não existe uma sala de informática que comporte todos os alunos simultaneamente, torna-se necessário que as atividades sejam realizadas fora do horário de aula;

- $\quad$ receio de que os detalhes técnicos ocupassem mais tempo e tivessem mais destaque do que o conteúdo cartográfico do exercício, particularmente para os alunos que não possuiam afinidade com a operação dos computadores.

Este último tópico é tratado com propriedade na obra intitulada "A educação na era da Internet: professores e aprendizes na web", coordenada por Nilton Santos.

Na introdução, o autor afirma: quando uma tecnologia surge, ela parece substituir a prática anterior, ela tende a ganhar mais importância do que o que se faz com ela. Quando a tecnologia está amadurecida e consolidada, ela parece desaparecer e se tornar invisivel, já que se incorpora à dinâmica do cotidiano (o qual ela também modificou), e a maneira de utilizá-la e o resultado da sua ação tendem a ganhar destaque sobre a tecnologia propriamente dita, que passa a ser parte do ambiente (SANTOS, 2000: 21).

No que se refere aos aspectos específicos do uso dos SIGs em sala de aula, ponderou-se sobre os principais itens citados no artigo de KEMP et al. (1998):

- $\quad$ percentual significativo dos conhecimentos tecnológicos sobre informações geográficas fica obsoleto em prazos inferiores a 6 meses;

- os produtos, serviços e idéias, relativos às informações geográficas, surgem em volume e velocidade muito maiores do que os usuários conseguem assimilar;

- $\quad$ é impossivel, para um professor, permanecer atualizado sobre as novidades da sua área de estudo e, simultaneamente, manter seu material didático no mesmo nível;

- a manutenção da qualidade do ensino de SIG fica comprometida diante do aumento do número de estudantes ou por conta do decréscimo ou manutenção dos orçamentos das instituições de ensino e pesquisa.

\section{A atividade didática usando um SIG na Internet}

Esse exercício representa uma das oito tarefas exigidas durante o curso de Introdução à Cartografia, ministrado no primeiro semestre do curso de Geografia da Faculdade de Filosofia, Letras e Ciências Humanas da Universidade de São Paulo (FFLCH-USP). A idéia central que norteou sua elaboração foi a de mostrar a aplicação de recursos tecnológicos na área da Cartografia, expondo seus potenciais e limites. Parte dos alunos ingressa no curso de Geografia com grandes expectativas, mas com pouco conhecimento em relação ao geoprocessamento. Por isso, torna-se importante esclarecer suas funções, relações, pré-requisitos e limitações. 
Essa atividade permitiu também inter-relacionar o conteúdo das disciplinas obrigatórias - Introdução à Cartografia, Cartografia Sistemática e Cartografia Temática -, ressaltando a necessidade de compreensão dos conceitos fundamentais (escala, sistema de coordenadas, projeção, formas de representação, variáveis visuais etc.) antes da utilização de qualquer sistema de informação geográfica.

\section{SpringWeb}

A opção pelo uso da Internet como meio de manipulação de dados geográficos decorreu da disponibilidade de recursos, fruto da tendência atual da geração dos SIGs, e do interesse pessoal do docente na exploração do potencial da rede. 0 SpringWeb foi escolhido porque é gratuito e de fácil implementação.

De acordo com a Divisão de Processamento de Imagens do Instituto Nacional de Pesquisas Espaciais (INPE), o SpringWeb 3.0 é um aplicativo escrito na linguagem de programação Java, que faculta a manipulação e a visualização de dados geográficos por meio da Internet. Permite que o usuário utilize algumas funções básicas de um SIG - consultas, mapas temáticos, zoom, impressão - usando apenas um navegador da Internet ${ }^{6}$ (ex: Explorer ou Netscape), sem que haja a necessidade de instalação do software SIG e da base de dados no computador utilizado.

0 dados do SpringWeb estão no formato GeoBR, sigla que significa Intercâmbio de Dados Geográficos no Brasil. 0 arquivo nesse formato é codificado em $\mathrm{ASCl}^{7}$, que garante fácil legibilidade e robustez. Contém, basicamente, dois tipos de linhas:

- palavras-chave: que identificam os diferentes tipos de componentes do formato;

- dados: expressos em coordenadas geográficas e atributos descritivos.

De acordo com LIMA (2002), a proposta do formato GeoBR incorpora inovações em relação aos formatos existentes, pois inclui todas as informações geográficas relevantes, minimiza as redundâncias e oferece uma forma simples de intercâmbio nos níveis sintático e semântico.

Embora os dados sejam do mesmo tipo dos formatos genéricos de intercâmbio (ex.: DXF do AutoCad, E00 do Arclnfo e MIF do Maplnfo), o formato GeoBR se diferencia por causa de duas características: a inclusão de metadados e o uso de um modelo genérico de informação contextual sobre 0 dado que está sendo transmitido ${ }^{8}$.

\section{Características do exercício}

Solicitou-se que os alunos respondessem questões e produzissem cartogramas sobre as caracteristicas dos municipios do estado de São Paulo. As informações sobre área $\left(\mathrm{km}^{2}\right)$, ano de fundação e população (Censo de 1991) de cada um dos 645 municípios do estado de São Paulo, fornecidos pelo Instituto Brasileiro de Geografia e Estatística (IBGE, situação de 1997), foram inseridas no servidor do Departamento de Geografia, de forma que o mapa e a tabela pudessem ser consultados e visualizados através do SpringWeb (ver exemplo da interface da fig. 1).

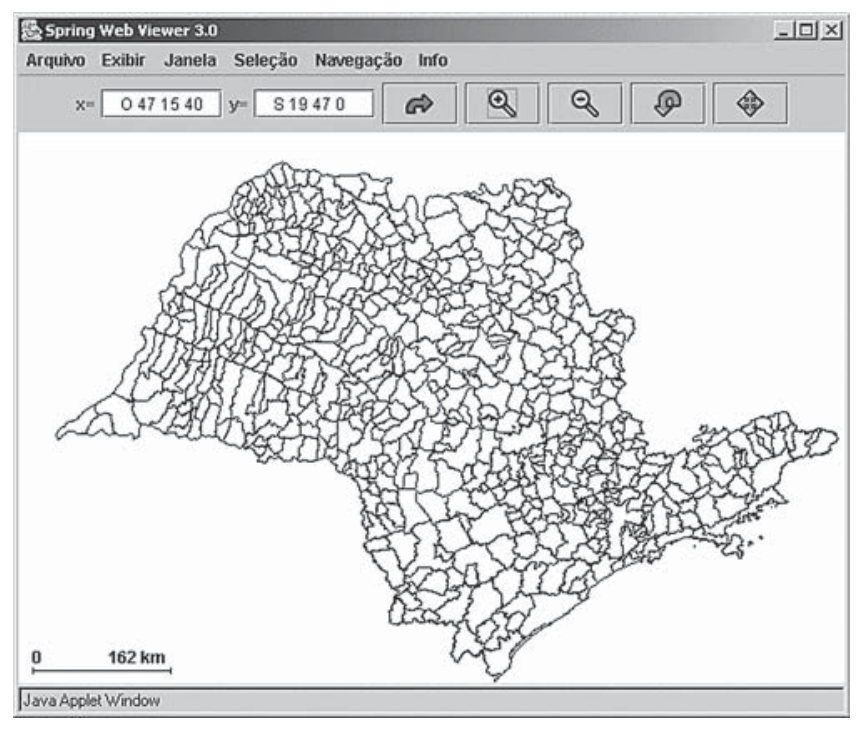

Figura 1 Interface do SpringWeb

0 conteúdo das questões resumiu-se à transformação da escala gráfica em numérica, elaboração de cartograma com figuras geométricas proporcionais, produção de mapas temáticos com número de classes e cores distintas, e elaboração de consultas estruturadas ao banco de dados (ex.: população 35.000 e área < 700).

As condições previamente estabelecidas, para a execução da atividade, foram:

- $\quad$ o uso do software SpringWeb, desenvolvido pelo INPE', disponivel no site do departamento (www.geografia. fflch.usp.br), nas opções graduação, apoio didático, mapa de SP;

- uso de banda larga, isto é, de acesso rápido à Internet (a

6 É possivel encontrar na literatura os termos web gis e gis on-line para caracterizar esta função.

7 American Standard Code for Information Interchange.

8 Ver maiores detalhes em: http://www.dpi.inpe.br/teses/lima/dissertacao_lima.pdf (acesso em 08 mar. 2003).

9 Consultar http://www.dpi.inpe.br (acesso em 22 jan. 2003). 
velocidade deve ser igual ou maior a $128 \mathrm{kbps})$. A velocidade de acesso, via telefone, da maioria dos modens é de 56 kbps. Embora não impeça a realização do exercício, torna o processo extremamente vagaroso; - $\quad$ instalação do plug-in Java, versão 1.3 .0 (jre1_3_0win.exe, com 6 mbytes), antes do primeiro acesso ao programa (somente uma vez em cada equipamento);

- os alunos deveriam efetuar o exercício fora do horário de aula. Embora sua realização pudesse ocorrer a partir de qualquer equipamento, foi sugerido o uso da sala pró-aluno (sala de informática de uso exclusivo dos alunos) ou do laboratório de Cartografia, que atendem às especificações descritas anteriormente.

Os critérios para a avaliação do exercício foram as notas dos trabalhos, realizados em grupos de no máximo quatro integrantes, a quantidade de dúvidas dos alunos, esclarecidas pelo professor e pelo monitor, e os relatos orais sobre o nível de dificuldade e de interesse, no ato da entrega do exercício.

Do total de 73 alunos, cursos diurno e noturno, a nota média dos grupos foi 7,2 e a moda 7,5 (avaliação de zero a dez). A nota mais baixa foi 3,5 e a mais alta, 9,5. As dúvidas mais freqüentes foram sobre a instalação do plug-in, para os alunos que usaram o programa fora dos locais sugeridos (sala pró-aluno e Laboratório de Cartografia) e sobre a função "salvar mapa", presente no menu superior, que não funciona.

É muito importante considerar esse último aspecto, pois impediu que os alunos executassem o exercício em um equipamento e imprimissem os mapas em outro. Como um dos locais sugeridos para a execução do trabalho, a sala pró-aluno, só possui impressora preto-e-branco (P\&B), e essa função "salvar mapa" não estava habilitada, parte dos alunos não conseguiu transportar o resultado do trabalho para outro equipamento, conectado a uma impressora colorida. Essa limitação determinou o percentual, de aproximadamente $20 \%$, de grupos que entregaram o trabalho impresso em P\&B. Embora fosse solicitado que os alunos explorassem as variações de cor nas representações gráficas, os mapas em tons de cinza foram aceitos sem decréscimo de nota.

Os relatos verbais sobre o interesse e dificuldades na realização do exercício, no ato da sua entrega ao professor, indicam que:

- $10 \%$ dos grupos gostaram muito da atividade e não tiveram dificuldades;

- $55 \%$ mencionaram como fato positivo a inovação e relataram dificuldades pontuais em determinadas etapas;

- $\quad 20 \%$ não responderam;

- $15 \%$ não gostaram do tipo de exercício e mencionaram grandes dificuldades para realizar a tarefa.

\section{Considerações finais}

Essa experiência com a Internet permitiu verificar o seu potencial de uso em sala de aula, mostrar seus limites, reforçar a importância dos conceitos básicos da Cartografia e ilustrar a relação da Cartografia com as disciplinas correlatas.

Desse ponto de vista, a utilização do SpringWeb se mostrou satisfatória, pois permitiu realçar visualmente as variações de escala, através das opções zoom (+ ou -), exercitar a transformação de escala gráfica para numérica, utilizar figuras geométricas proporcionais e explorar a variação do número de classes e a seqüência de cores nos cartogramas. Entretanto, o tempo gasto com os procedimentos técnicos da manipulação foi maior do que o esperado, mesmo com a suposição prévia de heterogeneidade de conhecimento de informática dos alunos.

A instalação do plug-in, a impossibilidade de transportar os arquivos (os mapas) e a quantidade de passos do roteiro de atividades consumiram atenção e tempo demasiados. Isso está diretamente ligado ao nível de familiaridade do aluno com o uso do computador. 0 tempo utilizado para compreender as etapas e os procedimentos aumenta diretamente em função da dificuldade de operação do computador, mas deve ser minimizada com o aperfeiçoamento da interface gráfica do SpringWeb.

Para os estudantes que não tinham nenhum contato prévio com computadores, esses objetivos mencionados não foram plenamente atingidos, pois as tarefas acabaram ficando ao encargo dos integrantes do grupo que já tinham essa habilidade, ou foram repetidas mecanicamente, após instrução dos colegas. Para a minoria do extremo oposto, que possui grande habilidade com as máquinas, não só as etapas foram realizadas sem nenhuma ajuda, como foi possível auxiliar os grupos com dúvidas. Os alunos com habilidade mediana, após esclarecimentos iniciais, conseguiram realizar o exercício com facilidade.

As principais vantagens de uso desse aplicativo no suporte às atividades didáticas do curso de Introdução à Cartografia são:

- $\quad$ possibilidade de trabalho fora do horário e local de aula. Esse aspecto é extremamente importante quando não houver infra-estrutura capaz de comportar todos os alunos (sala de informática equipada e funcionário para controlar o acesso fora do horário de aula);

resgate dos conceitos básicos de Cartografia. Foi possível, também, despertar o interesse sobre o conteúdo das disciplinas obrigatórias correlatas (Cartografia Sistemática e Temática) e optativas (Estágio Supervisionado em Geoprocessamento e Teoria e método em SIG); 
- $\quad$ gratuidade do software SpringWeb. Este aspecto permite que o investimento em software SIG seja eliminado;

- diminuição do investimento com hardware, pois qualquer equipamento que possua um navegador da Internet (ex: Explorer, Netscape etc.) pode ser utilizado. Como não há necessidade de instalação de software SIG no equipamento utilizado, não existe especificação mínima para os computadores;

- $\quad$ o desempenho dos alunos, mensurado pelas notas (média 7,2), foi considerado bom e superou a expectativa inicial. A participação, medida pelo número e pelo tipo de perguntas, foi considerada razoável. 0 nível de dificuldade foi considerado elevado para 15\% dos alunos.

As desvantagens mais significativas do uso do software SpringWeb em sala de aula, são:

- $\quad$ necessidade de aperfeiçoamento do SpringWeb (versão 3.0). Um número considerável de alunos relatou que a função "salvar mapa", presente no menu superior, não funciona. A baixa velocidade de resposta aos comandos e as circunstâncias nas quais o programa deixa de funcionar ("trava"), também foram mencionadas;

- $\quad$ exigência de acesso à Internet com conexão rápida (=> 128 kbps);

- $\quad$ obrigatoriedade de instalação do programa Java (plug-in);

- a rapidez de assimilação e realização da tarefa esteve, evidentemente, associada à heterogeneidade dos conhecimentos de informática dos alunos;

- demanda de conhecimento mínimo de informática. É importante, antes de reproduzir essa experiência, verificar se o nivel de conhecimento de informática dos alunos é compativel com o exercício. A utilização de editor de texto é um indicador, nesse caso, de conhecimento mínimo;

- $\quad$ necessidade de impressora colorida.
Embora o processo de integração de dados ainda esteja num estágio inicial, por conta da complexidade de padronização, existem várias iniciativas, como o GeoBR, para a criação de formatos de intercâmbio de dados. 0 desenvolvimento do SpringWeb, já na versão 3.0, é uma mostra inequívoca de que a Internet se tornará um meio essencial de acesso aos dados geográficos.

A importância de seu uso é ratificada por iniciativas de instituições governamentais brasileiras. Os exemplos que se assemelham, de maior destaque, são: o servidor de mapas do $I B G E$, que fornece informações sobre os municípios brasileiros $^{10}$ e o site da Embrapa, que oferece um mosaico de imagens de satélite, em variadas escalas, de todo o pais ${ }^{11}$.

Sua utilização torna-se ainda mais promissora se forem consideradas as seguintes tendências: de aumento da capacidade de manipulação de computadores - por parte dos alunos -, de aperfeiçoamento das interfaces gráficas e conseqüente diminuição da complexidade dos procedimentos técnicos e de minimização dos custos de acesso rápido à Internet.

Além disso, deve-se destacar a quantidade de iniciativas de desenvolvimento de programas gratuitos ${ }^{12}$, a importância da possibilidade de compartilhar o exercício elaborado com outros interessados (professores, alunos etc.) ${ }^{13}$ e a introdução de procedimentos que poderão ser úteis no cotidiano dos estudantes no mercado de trabalho.

Dessa forma, considera-se que os SIGs utilizados via Internet possuem um elevado potencial para melhorar as formas de consulta dos dados geográficos e de proporcionar um significativo aumento de alternativas para o ensino de Cartografia.

\section{Agradecimentos}

A Nabil Alameddine, pelo apoio nas atividades da disciplina de Introdução à Cartografia, em 2002.

10 Disponivel em http://www.ibge.gov.br (Acesso em 7 fev. 2003).

11 Disponivel em http://www.cdbrasil.cnpm.embrapa.br (Acesso em 25 mar. 2003).

12 Disponivel em www.freegis.org (Acesso em 26 jan. 2003).

13 A atividade pode ser utilizada, sem nenhuma restrição, por meio do endereço do site do Departamento de Geografia da Faculdade de Filosofia, Letras e Ciências Humanas da USP (www.geografia.fflch.usp.br opções: graduação, apoio didático, mapa de SP). 
QUEIROZ FILHO, A. P. (2002) Webgis: example of application in college. Revista do Departamento de Geografia, n.15, p. 115-122.

\begin{abstract}
This work tells the use of SpringWeb software in the course of Introduction to the Cartography, given in the first semester of 2002 to the students of the Department of Geography (University of São Paulo), and approaches the main aspects of the integration of data that give support to it. The possibilities to outside work of the schedule and place of lesson, to associate the basic concepts of Cartography and to minimize the investment in software and the hardware are considered the main advantages of this application. The disadvantages is relate to the heterogeneous knowledge of computer science of the students, necessity of use of broad band to have access the exercise and requirement of installation, in the first access, of one plug-in (Java program).
\end{abstract}

Key words: Geographic Information Systems (GIS); Internet; Cartography; Data integration.

Recebido em 10 de março de 2003, aceito em 16 de junho de 2003.

\section{Referências bibliográficas}

CÂMARA, G.; MEDEIROS, C.B.; CASANOVA, M.A.; HERMELY, A.; MAGALHÃES, G. (1996) Anatomia de Sistemas de Informação Geográfica. Campinas, Instituto de Computação/UNICAMP, $197 \mathrm{p}$.

GOODCHILD, M.; EGENHOFER, M.J.; FEGEAS, R. (1997) Interoperating GISs: Panel on computational implementations of geographic concepts. In: INTERNATIONAL CONFERENCE \& WORKSHOP ON INTEROPERATING GEOGRAPHIC INFORMATION SYSTEMS. Santa Bárbara, 1997. Anais eletrônicos. Disponível em <http://www.ncgia.ucsb.edu/conf/interop97/ report.html\#section $1>$. Acesso em 15 jan. 2003.

GREEN, D.; BOSSOMAIER, T. (2002) Online GIS and Spatial metadata. New York, Taylor \& Francis. $222 \mathrm{p}$.

KEMP, K.; REEVE, D. E; HEYWOOD, I. (1998) Report of the International Workshop on Interoperability for GIScience Education. In: INTERNATIONAL WORSHOP ON INTEROPERABILILITY FOR GISCIENCE EDUCATION. The Netherlands, 1998. Anais eletrônicos. Disponivel em $<$ http://www.ncgia.ucsb.edu/ige98/report/ige98.pdf $>$. Acesso em 26 fev. 2003.
LIMA, P. (2002) GeoBR Intercâmbio de dados espaciais: modelos, formatos e conversores. São José dos Campos, $1 \mathrm{v}$. Dissertação (Mestrado) - Instituto Nacional de Pesquisas Espaciais. Disponivel em <http://www.dpi.inpe.br/teses/

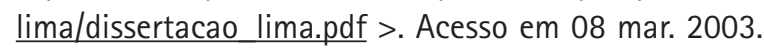

NCGIA. National Center for Geographic Information and Analysis. (1996) Iniciative 19. In: GIS AND SOCIETY: The social implications of how people, space and environment are represented in GIS. Minnesota, 1996, Anais eletrônicos. Disponivel em <http:/l www.geo.wvu.edu/i19/> Acesso em 06 mar. 2003.

SANTOS, N. (coord.) (2000) A educação na era da Internet: professores e aprendizes na web. Rio de Janeiro, Editora Clube do Futuro. $156 \mathrm{p}$.

WATERS, N. (1997) Marks the end of the GIS revolution. GIS World. v.10, n.1, p.71. 